\title{
Estudo de potenciais doadores de córnea no Hospital de Clínicas da UFPR
}

\section{Study of cornea potential donors at the Clinical Hospital of UFPR}

Glauco Henrique Reggiani Melloํㅜ, Thais Martini Massanares², Guilherme Barroso Guedes ${ }^{3}$, Daniel Wasilewski ${ }^{4}$, Hamilton Moreira ${ }^{5}$

\begin{abstract}
ReSUMO
Objetivo: Quantificar os potenciais doadores de córnea nas diferentes unidades hospitalares do Hospital de Clínicas da Universidade Federal do Paraná - UFPR. Métodos: Foram analisados 369 prontuários de pacientes que foram a óbito nos meses de abril a julho de 2005 no HC-UFPR. No prontuário de cada paciente foram avaliados: evolução clínica, CID-10 e unidade hospitalar do óbito. Para ser considerado potencial doador (PD) utilizou-se como critérios de exclusão os tópicos citados no Manual de Transplantes ( $2^{\mathrm{a}}$ edição - 2004) da Secretaria Estadual da Saúde do Estado do Paraná. Os PD foram agrupados em: data do óbito, tipo de internamento e unidade hospitalar. Analisamos a quantidade de PD e total de óbitos. Resultados: Dos 369 prontuários analisados, foram considerados potenciais doadores (PD) 70 pacientes (18,97\% do total de prontuários analisados) As unidades: Centro de Terapia Intensiva, Centro de Terapia Semi e Pronto Atendimento Adulto foram responsáveis por 62,8\% dos PD. Já a Neurocirurgia, Emergência Cárdio e UTI Cirurgia Cardíaca apresentaram uma relação de PD por óbito superior a $60 \%$. Conclusão: Nesse estudo constatou-se que tanto as unidades com grande número de PD como aquelas com uma grande relação PD por óbito são locais de escolha para uma intervenção com o intuito de aumentar o número de doações em nosso meio.
\end{abstract}

Descritores: Doadores de tecidos; Hospitais gerais; Transplante de córnea

\footnotetext{
${ }^{1}$ Research Fellow Cleveland Clinic - USA;

${ }^{2}$ Acadêmica do $6^{\circ}$ ano de Medicina do Hospital de Clínicas da Universidade Federal do Paraná - UFPR - Curitiba (PR), Brasil;

${ }^{3}$ Research Fellow Yale University - Yale - USA;

${ }^{4}$ Preceptor de Residência em Oftalmologia da Universidade Federal do Paraná - UFPR - Curitiba (PR), Brasil;

${ }^{5}$ Professor Adjunto do Departamento de Oftalmologia/Otorrinolaringologia do Setor de Ciências da Saúde do Hospital de Clínicas da Universidade Federal do Paraná - UFPR - Curitiba (PR), Brasil;

Trabalho realizado no Departamento de Oftalmologia/Otorrinolaringologia do Setor de Ciências da Saúde do Hospital de Clínicas da Universidade Federal do Paraná - UFPR - Curitiba (PR), Brasil.

Declaração de interesse e ineditismo:

Cada autor declara que não possui interesse financeiro no desenvolvimento ou marketing dos (instrumentos, medicação) referidos no estudo. Declaram ainda o caráter inédito do texto, sendo esta, uma vez aprovada a primeira publicação do artigo.
}

Recebido para publicação em: 24/2/2010 - Aceito para publicação em 14/7/2010 


\section{INTRODUÇÃO}

$\mathbf{0}$ transplante de córneas é um dos procedimentos cirúrgicos mais importantes da oftalmologia pelo que representa a recuperação visual de um indivíduo na sociedade ${ }^{(1)}$. A indicação de ceratoplastia penetrante tem aumentado muito nos últimos 30 anos e está entre as cirurgias de transplante mais realizadas ${ }^{(2,3)}$. $\mathrm{O}$ aumento na indicação de ceratoplastia penetrante se deve a alguns fatores, entre eles o aumento da faixa etária da população, melhor seleção do tecido doador e novas técnicas operatórias que permitem a realização de cirurgias em situações consideradas antes inoperáveis ${ }^{(4)}$.

Apesar de a ceratoplastia penetrante ter se tornado o procedimento mais comum em relação aos transplantes ${ }^{(5,6)}$, ainda existem milhares de pessoas no Brasil à espera de uma córnea ${ }^{(7)}$. Este quadro impressiona, pois o tempo de espera para a cirurgia algumas vezes supera a sobrevida do paciente candidato ao transplante ${ }^{(7,8)}$. No primeiro semestre de 2009, a lista de espera no Brasil superava 22.727 pacientes, só no estado do Paraná são mais de 1.322 pacientes a espera de um transplante ${ }^{(9)}$. Neste mesmo período de 2009 foram implantadas no Brasil 6.151 córneas, destas 507 no estado do Paraná ${ }^{(10)}$

Hoje ainda uma das maiores limitações para o aumento dos transplantes é o número de doações ${ }^{(7,11-13)}$. Até Agosto de 2005 haviam sido captadas no Hospital de Clínicas da UFPR apenas 12 córneas ${ }^{(10)}$.

Com o interesse de conhecer melhor a realidade de nosso serviço quantificou-se o número de potenciais doadores (PD) de córnea nas diferentes unidades do Hospital de Clínicas da UFPR.

Esse trabalho tem por objetivo conhecer melhor o perfil de potenciais doadores (PD) de córnea de nosso serviço e quantificar os mesmos em relação as diferentes unidades do Hospital de Clínicas da UFPR.

\section{MÉTODOS}

Estudo descritivo da incidência de PD foi realizado de forma retrospectiva no Hospital de Clínicas (HC) da Universidade Federal do Paraná (UFPR). Foram analisados 369 prontuários de 369 pacientes que foram a óbito no período de abril a julho de 2005 no HC da UFPR.

No prontuário de cada paciente foram avaliados: a evolução clínica, CID-10 e unidade hospitalar do óbito. Foram considerados não doadores pacientes que apresentaram algum critério de exclusão citado no Manual de Transplantes do Paraná ${ }^{(14)}$.

Os PD foram categorizados quanto à unidade hospitalar do óbito.

Os seguintes critérios de exclusão foram obser- vados: tecidos retirados de doadores cuja causa mortis tenha sido: morte de causa desconhecida, hepatite viral aguda, septicemia, raiva, aids, doença de CreutzfeldtJakob, panencefalite subaguda esclerosante, rubéola congênita, linfomas ativos disseminados, leucemias, síndrome de Reye, encefalite viral ativa ou de origem desconhecida ou encefalopatia progressiva, leucoencefalopatia multifocal progressiva, doença neurológica de diagnóstico indeterminado, endocardite (bacteriana ou fúngica) ativa. Doadores com doenças intrínsecas do olho: retinoblastoma, tumores malignos do segmento anterior ocular ou adenocarcinoma do olho (de origem primária ou metastática), inflamação ativa: ocular ou intraocular (conjuntivite, esclerite, irite, uveíte, coroidite, retinite), desordens congênitas ou adquiridas (cicatriz central da córnea, ceratocone, ceratoglobo). Doadores com testes sorológicos recentes para HBs AG,Anti-HBc, Anti-HCV,Anti-HIV-1 a Anti-HIV-2.

\section{Resultados}

Dos 369 prontuários analisados foram considerados potenciais doadores 70 pacientes $(18,97 \%$ do total).

Nos quatro meses de estudo, o número de PD mensal variou de 17 a 21. Já o número de pacientes que foram a óbito variou de 85 a 98 no período em análise (Gráfico 1).

Os resultados apresentados mostram a incidência de PD em relação ao número de óbitos totais nas diferentes unidades hospitalares.

De acordo com o tipo de internamento, dos 70 PD apenas um internou de forma eletiva $(1,42 \%)$. Por outro lado, 69 PD (98,58\%) internaram por motivo de emergência.

As unidades hospitalares que apresentaram maior número de PD fazem parte da emergência da Clínica Médica: Centro de Terapia Intensiva com 21,4\% dos PD, Centro de Terapia Semi-Intensiva com $21,4 \%$ dos PD e Pronto Atendimento Adulto com 20\% dos PD. Outras unidades encontram-se citadas na Tabela 1.

Foram analisadas também as unidades: Infectologia, Cirurgia Aparelho Digestivo, Cirurgia Urológica, Transplante Hepático, Nefrologia, Transplante de Medula, Emergência Pediátrica, Neonatologia, Cirurgia Ginecológica, Quimioterapia, Cirurgia Vascular, Unidade de Internação F, Transplante Renal, Neonato Risco Intermediário e Hematopediatria. Nestas, de um total de 47 óbitos no período, não houve PD.

Por outro lado, as unidades que apresentaram maior porcentagem de PD em relação ao seu número de óbito foram: Neurocirurgia com $75 \%$, Emergência Cardio com $60 \%$ e UTI Cirugia Cardíaca com 57,1\% (Tabela 1).

De acordo com a Secretaria Estadual da Saúde 


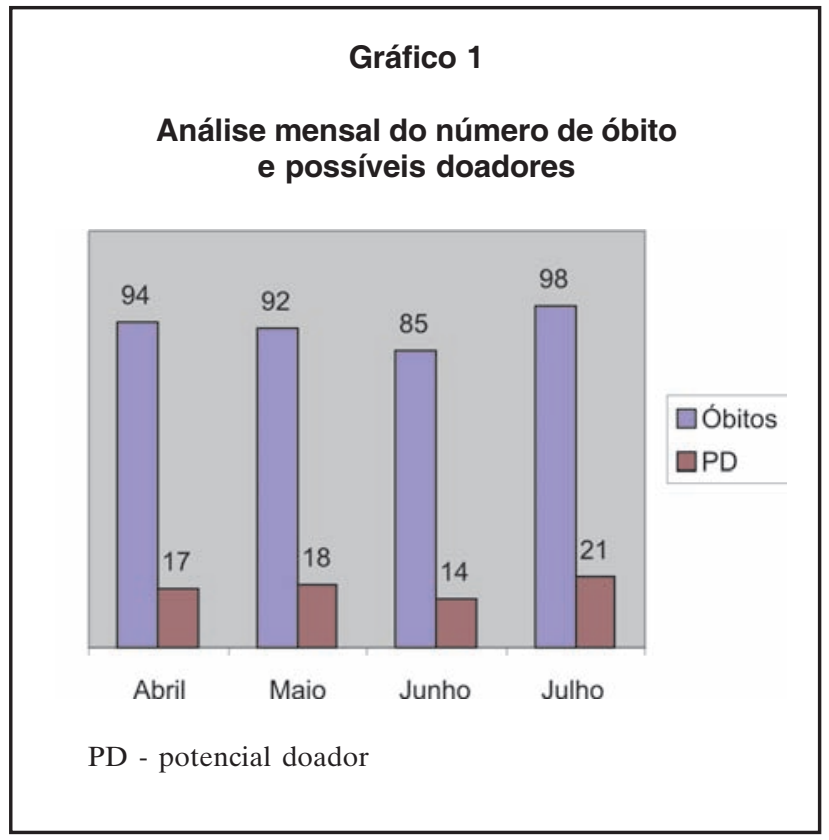

do Paraná, no período de estudo houve apenas uma captação de córnea no HC da UFPR.

\section{Dıscussão}

Sabe-se hoje que o transplante de córnea constitui uma alternativa terapêutica cada vez melhor para grande parte das doenças corneanas. No entanto, o número de transplantes de córnea está aquém do necessário. No nosso serviço, apenas uma córnea foi captada durante todo o período de estudo (4 meses). Na literatura, a principal barreira para a melhora deste panorama é a falta de doadores esclarecidos e, este parece ser um reflexo da desinformação por parte da população e da classe médica, bem como de obstáculos legais e religiosos ${ }^{(7,12,13)}$.

A influência da equipe médica no número de doadores de órgãos e tecidos foi levantada por muitos au-

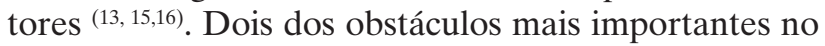
processo de doação foram a não procura de doadores e a falência na abordagem ${ }^{(17)}$.

Através do resultado obtido em nosso estudo estes podem ser os motivos de tão pouca captação de córneas no Hospital de Clínicas da UFPR. Foram identificados 70 potencias doadores em 4 meses com apenas uma captação, ou seja, 138 córneas potenciais que não foram utilizadas, $414 \mathrm{em} 1$ ano, o que representa $28 \%$ do número de pacientes na fila de espera do Paraná que poderiam ser submetidos ao transplante ainda este ano.

No nosso estudo, cerca de $87 \%$ dos PD de córnea encontraram-se em unidades de tratamento intensivo, concordando com a literatura em que $95 \%$ dos doadores
Tabela 1

\section{Análise das diferentes unidades hospitalares quanto ao número de óbitos, $\mathrm{PD}$ e relação dos PD pelos óbitos e PD pelo total de PD}

\begin{tabular}{lcccc}
\hline Unidade hospitalar & Óbitos & PD & $\begin{array}{c}\text { \% PD/ } \\
\text { Óbitos }\end{array}$ & $\begin{array}{c}\text { \% PD/ } \\
\text { PD total }\end{array}$ \\
\hline Centro de terapia intensiva & 83 & 15 & 18 & 21,4 \\
Centro de terapia semi & 72 & 15 & 20,8 & 21,4 \\
Pronto atendimento adulto & 53 & 14 & 26,4 & 20 \\
UTI cirurgia cardíaca & 14 & 08 & 57,1 & 11,4 \\
Neurocirurgia & 04 & 03 & 75 & 4,3 \\
Emergência neurológica & 09 & 03 & 33,3 & 4,3 \\
Emergência cárdio & 05 & 03 & 60 & 4,3 \\
Clinica médica masculina & 21 & 02 & 9,5 & 2,8 \\
Clinica médica feminina & 10 & 02 & 20 & 2,8 \\
UTI pediátrica & 21 & 02 & 9,5 & 2,8 \\
Policlínica & 03 & 01 & 33,3 & 1,4 \\
Cirurgia geral & 10 & 01 & 10 & 1,4 \\
UTI & 17 & 01 & 5,9 & 1,4 \\
\hline
\end{tabular}

PD - potencial doador; UTI - unidade de terapia intensiva

de córnea em potencial localizam-se nas unidades de terapia intensiva ${ }^{(16)}$.

A porcentagem média de $\mathrm{PD}$ no $\mathrm{HC}$ durante o período de análise foi de 18,97\% dos óbitos, com uma variação mensal de $16,5 \%$ a $21,4 \%$. Carrey et al. encontraram 17\%, Siminoff et al. 18,9\% e Geissler et al. $20,4 \%$ de taxa de doações de córnea ${ }^{(18,20)}$.

A análise dos dados indica as unidades intensivas como o melhor local para intervenção e aumento no número de captação de córneas (Tabela 1). A Clínica Médica é responsável pelas três unidades com maior número de PD: Centro de Terapia Intensiva, Centro de Terapia Semi e Pronto Atendimento Adulto. Nesses locais a relação de PD chega a 62,8\% do total.

Quando avaliada a relação $P D$ por óbito por unidade algumas apresentaram esse índice significativamente superior ao dos locais apontados para intervenção. No entanto, aquelas apresentaram número absoluto baixo de PD.

A necessidade de voluntários e profissionais médicos e não médicos trabalhando ativamente nos programas de captação de órgãos é um dos fatores essenciais para que se aumente o número de doadores ${ }^{(11)}$. A abordagem e a confiança na equipe médica levam a um aumento direto no número de doações ${ }^{(12)}$. Para este fim, como tem sido sugerido em vários estudos , torna-se necessária a criação de comissões e centrais de doação de órgãos, bem como a realização de campanhas de esclarecimento ${ }^{(11,12,16)}$.

Comparada com a publicidade que é dada a outros setores da medicina, o transplante de córnea não é muito divulgado, mas ele marca a diferença na qualidade de vida das pessoas que dele necessitam ${ }^{(21)}$. Esse fato 
deve ser entendido para que se tenha uma melhor informação, divulgação e motivação das pessoas e profissionais da área médica, para que a recuperação visual não tenha como limitante a falta de doadores ${ }^{(7)}$.

\section{Conclusão}

A partir desta análise, constatou-se setenta possíveis doadores no período de 4 meses, tendo apenas um efetivado a doação, havendo um claro desperdício de órgãos e acarretando uma longa fila de espera. Faz-se acreditar ser de suma importância um planejamento e intervenção para melhorar a captação de córneas para transplante nas unidades com um grande número de PD: Centro de Terapia Intensiva, Centro de Terapia Semi e Pronto Atendimento Adulto. Não descartando unidades com uma grande relação de PD por óbito como a: Neurocirurgia, Emergência Cárdio e UTI Cirurgia Cardíaca.

\section{Abstract}

Purpose: To quantify the potential cornea donor from the different units of Clinical Hospital of the UFPR. Methods: 369 records of patients who died from april until july of 2005 in the HC-UFPR were analyzed. The medical records of each patient had been evaluated: the clinical evolution, CID-10 and hospital unit of the death. To be considered potential donor $(P D)$ it was used as exclusion criteria the topics cited in the Manual of Transplants ( $2^{a}$ edition-2004) of the State Secretariat of the Health of the State of the Paraná. The PD had been grouped in: date of the death, type of internment and hospital unit. We analyze the amount of PD and total of deaths. Results: Of 369 analyzed records, potential donor (PD) had been considered 70 patients (18.97\% of the analyzed handbook total) the units: Center of Intensive Therapy, Center of Half Therapy and ER Adult had been responsible for $62, .8 \%$ of the PD. Already the neurosurgery, cardiac emergency and UTI cardiac surgery had presented a relation of $P D$ per death superior than $60 \%$. Conclusion: In this study we evidence that as much the units with great number of potential donor (PD) as those with a great relation $P D$ per death are local of choice for an intervention with intention to increase the number of donations in our way.

Keywords: Tissue donors; Hospital, general; Corneal transplantation

\section{ReFERÊNCIAS}

1. Arentsen JJ, Laibson PR. Surgical management of pseudophakic corneal edema: complications and visual results following penetrating keratoplasty. Ophthalmic Sug. 1982;13(5):371-3.
2. Acedo JT. Queratoplastias y queratoprótesis. 2a. ed. Barcelona: EdikaMed; 1992.

3. Belfort Júnior R. Ceratoplastias e ceratectomia. In: Belfort Júnior R, Kara-José N, editores. Córnea: clinica-cirurgica. São Paulo: Roca; 1996. p.493-504.

4. Lindiquist TD, McGlothan JS, Rotkis WM, Chandler JW. Indications for penetrating keratoplasty: 1980-1988. Cornea. 1991;10(3):210-6.

5. Freitas JAH, Soranz JE, Rovigatti Júnior V, Barbosa ML, Hirashima DE, Felberg S. Ceratoplastia penetrante: experiência de um ano de um banco de ohos vinculado a um Hospital escola. Rev Bras Oftalmol. 1997;56(10):749-54.

6. Report of the organ transplant panel. Corneal transplantation. Council on Scientific Affairs. JAMA. 1988;259(5):71922. Review.

7. Alves MR, Crestana FP, Kanatani R, Cresta FB, José NK. Doação de córnea: opinião e conhecimento de médicos intensivistas do Complexo Hospital das Clinicas da Faculdade de Medicina da Universidade de São Paulo. Rev Med (São Paulo). 1997;76(6):315-9.

8. Nenno C, Abel R Jr. Cornea transplant statistics in the United States. Refract Corneal Surg. 1991;7(6):467-8.

9. Brasil. Ministério da Saúde. Transplantes [internet]. [citado 2010 Apr 21]Disponível em: http://dtr2001.saude.gov.br/ transplantes/

10. Secretaria Estadual da Saúde do Paraná [Internet]. [citado 2010 Apr 21] Disponível em: www.saude.pr.gov.br/ Transplante/

11. Farge EJ, Silverman ML, Khan MM, Wilhelmus KR. The impact of state legislation on eye banking. Arch Ophthalmol. 1994;112(2):180-5.

12. Diamond GA, Campion M, Mussoline JF, D'Amico RA. Obtaining consent for eye donation. Am J Ophthalmol. 1987;103(2):198-203.

13. Loewenstein A, Rahmiel R, Varssano D, Lazar M. Obtaining consent for eye donation. Isr J Med Sci. 1991;27(2):79-81.

14. Brasil. Paraná (Estado). Secretaria Estadual da Saúde. Central Estadual de Transplantes. Manual de transplantes. 2a ed. Paraná: Secretaria Estadual da Saúde; 2004.

15. Ishay R. Eye donation-how to maximize procurement. Isr $\mathbf{J}$ Med Sci. 1991;27(2):89-91. Comment on: Isr J Med Sci. 1991;27(2):79-81.

16. McGough EA, Chopek MW. The physician's role as asker in obtaining organ donations. Transplant Proc. 1990;22(1):267-72.

17. Mack RJ, Mason P, Mathers WD. Obstacles to donor eye procurement and their solutions at the University of Iowa. Cornea. 1995;14(3):249-52.

18. Carrey I, Hudel Y, Salame N, Queguigner F, Maux R, Delbosc B. [Procurement of corneas: analysis of inventory of hospital coordination. Prospective study at the Besançon Hospital University during the course of one year] J Fr Ophtalmol. 2000;23(10):996-1000. French.

19. Siminoff LA, Arnold R, Miller DS. Differences in the procurement of organs and tissues by health care professionals. Clin Transplant. 1994;8(5):460-5.

20. Geissler A, Paoli K, Maitrejean C, Durand-Gasselin J. Rates of potential and actual cornea donation in general hospital: impact of exhaustive death screening and surrogate phone consent. Transplant Proc. 2004;36(10):2894-5.

21. Rodrigues AM, Sato EH. Conhecimento e atitude da população do Hospital São Paulo sobre doação de córneas. Arq Bras Oftalmol. 2002;65(6):637-40.

Endereço para correspondência:

Glauco Henrique Reggiani Mello

Rua Lamenha Lins, ${ }^{\circ} 447$ apto. 21

CEP 80250-020 - Curitiba (PR), Brasil

e-mail: glaucohrm@gmail.com 